\title{
Editorial
}

\section{Reflections on the year that was 2020}

\section{Cowin AJ}

For referencing Cowin AJ. Reflections on the year that was 2020. Wound Practice and Research 2020; 28(4):153.

DOI https://doi.org/10.33235/wpr.28.4.153

2020 is the 20th year of the third millennium, the 20th year of the 21 st century and the first year of the 2020s decade. 2020 has also been a highly disruptive year that has been heavily defined by the COVID-19 pandemic; which has led to global, social and economic disruption, mass cancellations and postponements of events, worldwide lockdowns, worldwide protests and the largest economic recession since the Great Depression.

2020 was also designated as the Year of the Nurse and Midwife by the World Health Organisation. It was the year that our clinicians were never more valued, trusted and appreciated by every member of the public. As our first line of defense, our nurses, doctors and allied health staff protected the most vulnerable members of our communities, helping to keep them safe from the insidious disease that is COVID 19.

While we can look back at all things that we could not achieve or do in 2020, such as the WUWHS that was to bring thousands of wound care clinicians and researchers together in Abu Dhabi to learn and upskill through collegiality and friendship, or our own Wounds Australia national conference that could not take place, we can also value the things that we have learnt and achieved despite the restrictions.

2020 was the year that we learnt how to use Zoom, attend virtual seminars and hold meetings with people that we normally would not see. Virtual conferences and meetings enabled many people who would not normally be able to attend international and national conferences to gain knowledge and learning in their own homes and workplaces. We learnt how to work safely, effectively and efficiently from home and how to navigate using QR codes and PPE when needed within our workplaces.

With the journal, we continued to receive high quality submissions from authors from within Australia but also increasingly from international authors, highlighting our continuing global reach and the decreasing barrier of distance. One such highlight is being able to announce the winners of the 2019 Coloplast literary awards. We had hoped

Prof Allison J Cowin

Editor Wound Practice and Research to be able to present our winners at the Wounds Australia conference, but this was not to be. However, they have all received their winners' and runners'-up certificates as well as the winners receiving a cheque for $\$ 1000$ to be spent on furthering their wound careers. We sincerely thank Coloplast for their continuing support of these awards that recognise the quality and impact of our authors.

\section{Original Research Article \\ Winner}

Syed Sultan Beevi and colleagues: In vitro healing efficacy of stem cell secretome and cord blood platelet lysate on a chronic wound model (Secunderabad, India).

Runner-up

Cintia M Martinez-Garduno and colleagues: The Surgical Patients' Pressure Injury Incidence (SPPII) study: a cohort study of surgical patients and processes of care (St Vincent's Health Australia (Sydney)).

\section{Literature Review/Clinical Practice}

Winner

Christina Parker and colleagues: Methods for chronic wound research - A scoping systematic review of the recommendations, guidelines and standards (QUT, QLD).

Runner-up

Robyn Rayner and colleagues: Defining age-related skin tears: a review (Curtin University, WA).

\section{Case Study/Series}

\section{Winner}

Sue Randall and colleagues: Getting lower leg ulcer care evidence into primary health care nursing practice: a case study (University of Sydney, NSW).

\section{Runner-up}

Leonardo Cordova and colleagues: Negative pressure wound therapy in the management of complex lower limb wounds: a case series highlighting outpatient care with small single-use devices (Frankston Hospital VIC).

So, while 2020 is not a year that we will look back on with any great fondness, there have still been highlights and events that we can appreciate while we all hurry forward into 2021. 